\title{
A structured genetics rotation for pediatric residents: an important educational opportunity
}

\author{
RaeLynn Forsyth, MD ${ }^{1}{ }^{1}$, Weiyi Mu, ScM, CGC ${ }^{2}$, Laura Gibson, C-TAGME², Janet R. Serwint, MD³, \\ Nicole Shilkofski, MD, Med ${ }^{3}$ and Joann Bodurtha, MD, $\mathrm{MPH}^{1}$
}

Purpose: As the integral role of genetics in health and disease becomes increasingly understood, pediatricians must incorporate genetic principles into their care of patients. Structured exposure to genetics during residency may better equip future pediatricians to meet this goal.

Methods: Pediatric interns in the Johns Hopkins pediatric residency program have the option to spend one week immersed in clinical genetics by attending outpatient clinics and seeing inpatient consults. A pretest assessing clinical genetics knowledge is given before the rotation and compared with an identical post-test. Interns have a "scavenger hunt" to introduce genetic resources useful to pediatricians and complete a logbook of patient experiences. An evaluation is completed at the end of the rotation.

Results: Since the selective started in July 2016, 50 interns have participated. Average pretest score was 2.5/5 compared with a posttest score of $4.3 / 5, p<0.0001$. Interns saw on average ten patients and four different diagnoses. Overall evaluation was 4.4 on a 5 point scale, 5 being "excellent."

Conclusion: This experience suggests that a structured rotation in genetics provides pediatric interns with an opportunity to learn basic clinical genetics knowledge and skills and see patients whom they may otherwise not encounter during residency.

Genetics in Medicine (2020) 22:793-796; https://doi.org/10.1038/s41436019-0723-7

Keywords: pediatrics; clinical genetics; residency; medical education; genetics rotation

\section{INTRODUCTION}

As the integral role of genetics in health and disease becomes increasingly understood, physicians must gain familiarity with and incorporate medical genetics into primary care. ${ }^{1}$ The National Coalition for Health Professional Education in Genetics has published recommendations for core competencies in genetics for all health-care professionals, focusing on knowledge, skills, and attitudes. ${ }^{2}$ Despite this educational need, the Accreditation Council for Graduate Medical Education (ACGME) has no official requirements for pediatric residency programs to offer training in medical genetics. ${ }^{3}$ Recent studies have shown that pediatricians report low genetic literacy and lack of familiarity with family history taking and genetic testing, resulting in underutilized genetic testing and missed opportunities to establish a diagnosis in patients who meet clinical criteria for specific genetic diagnoses. $^{4-6}$

Structured exposure to genetics during residency may equip pediatricians with knowledge and skills that could improve patient care and their comfort level in caring for patients. One pediatric institution has shown that a required rotation in medical genetics during residency training increases awareness of the importance of medical genetics. ${ }^{7}$ Other training programs have tried a variety of initiatives to increase genetics competencies, such as a one-time web-based module for trainees in family medicine, a workshop at a national meeting for trainees in pathology, and a didactic course for trainees in dermatology. ${ }^{8}$ Educational opportunities focused on genetics for medical students, such as team-based learning activities, have also been described in the literature. ${ }^{9}$ This is the first report, however, that has qualitative and quantitative date to support the benefit of a rotation in genetics for pediatric trainees.

A two-week selective focused on outpatient medicine for firstyear pediatric residents (interns) was introduced to the pediatric residency training program at Johns Hopkins Hospital at the start of the 2016-2017 academic year. The term "selective" means that residents have different options to fulfill the rotation requirement, and the options for this particular selective are a subspecialty outpatient experience or a general pediatric outpatient experience. All interns who choose the subspecialty option spend one of their two weeks immersed in clinical genetics and spend the second week in a different subspecialty of their choice. The curriculum for the genetics portion of the selective was adapted from the medical student elective in genetics and tailored to meet the needs and objectives of a pediatric intern by giving them more in-depth clinical scenarios

\footnotetext{
${ }^{1}$ Department of Pediatrics and McKusick-Nathans Department of Genetic Medicine, Johns Hopkins University School of Medicine, Baltimore, MD, USA; ${ }^{2}$ McKusick-Nathans Department of Genetic Medicine, Johns Hopkins University School of Medicine, Baltimore, MD, USA; ${ }^{3}$ Department of Pediatrics, Johns Hopkins University School of Medicine, Baltimore, MD, USA. Correspondence: RaeLynn Forsyth (rforsyt2@jhmi.edu)
} 
on the scavenger hunt (described below in "Materials and Methods") and more autonomy in clinical encounters.

\section{MATERIALS AND METHODS}

\section{Scheduling}

Interns can be scheduled to do their selective at any time during their first year of residency. There are no prerequisites for the selective. Due to the availability of resources and clinical opportunities at our institution, up to two interns are scheduled on the genetics selective at one time.

\section{Pretest}

Prior to starting the selective, interns receive an email with materials for the selective described below and on the first day of the rotation, meet with a member of the genetics faculty to review learning objectives, and complete a pretest (Supplementary Appendix A). The pretest consists of five questions designed to assess knowledge of a broad array of genetics topics including appropriate work-up, resources, possible results of genetic testing, genetic counseling language, and features of a specific genetic condition. Interns are not automatically informed of the correct answers after taking the pretest, but clarifications in addition to answers to general questions regarding genetics topics of interest are provided upon request.

\section{Patient experiences}

Depending on the outpatient genetics schedule for each particular week, interns are exposed to different types of patients. Such patients could include those being seen in specialty clinics such as skeletal dysplasia clinic or connective tissue disease clinic, or those patients undergoing evaluation or follow-up in general genetics clinic for dysmorphic features or congenital anomalies, developmental delays or intellectual disability, cancer predisposition, metabolic conditions, or neurological disease. While in the clinic, interns work directly with an attending and/or genetic counselor from the Department of Genetic Medicine. Interns also see any inpatient consults for patients who could have otherwise been seen in outpatient clinic with the on-call genetics resident and attending. These most commonly are consults from the neonatal intensive care unit (NICU). Interns record all of their patient encounters in a logbook (Supplementary Appendix B) with details such as patient information, reason for visit, disease category from one of those described above, and setting of patient encounter. They also indicate their level of involvement with history taking, performance of physical exam, and drawing of the patient pedigree. Finally, they are requested to write at least one learning point from each encounter.

\section{Scavenger hunt}

In the downtime between clinics and patient encounters, interns are provided with a "scavenger hunt" (Supplementary Appendix C) with items to broaden their exposure to various genetic topics and conditions that they may otherwise not encounter on the selective. The scavenger hunt directs interns to commonly used online resources and provides references for them to review foundational articles in genetics and important clinical guidelines. Goals of the scavenger hunt include learning genetic counseling language and family history taking skills, reading about genetic testing such as chromosomal microarray and clinical exome sequencing, and using resources to learn about the evaluation and management of several genetic conditions. Resources that they are encouraged to use and familiarize themselves with include those that are useful for medical providers, such as the $\mathrm{OMIM}^{\circledR}$ database, the American College of Medical Genetics (ACMG) ACTion (ACT) sheets for newborn metabolic screening, and GeneReviews ${ }^{\circledR}$ (which contain summaries of many genetic syndromes). Interns compare these resources with those that have more patient-friendly language, such as Genetics Home Reference (which contains summaries for many genetic syndromes) and Baby's First Test for newborn metabolic screening. Articles that supplement the scavenger hunt provide overviews of general genetics topics including pedigree nomenclature, the evaluation of a dysmorphic infant, inborn errors of metabolism, informed consent for genetic testing, and clinical exome sequencing. Guidelines on the indications for a referral to genetics, the genetic evaluation of autism spectrum disorders, and the management of specific connective tissues diseases are also provided for review. A complete list of these references is available at the end of the scavenger hunt (Supplementary Appendix C).

\section{Other learning experiences}

Unless they are required to be in their own half-day pediatric continuity clinic, interns attend the weekly clinical conference held in the Department of Genetics. At this 1-hour conference, presentations can include learning points from recent inpatient consults, interesting updates on prior patients seen on consultation or in clinic, and review of guidelines for diagnosis and/or management of various genetic conditions. Most interns are also able to participate in the weekly didactic lectures given by faculty to the genetics residents. Interns also are invited to attend the weekly departmental journal club and other relevant conferences occurring during their time on the selective.

\section{Post-test}

On the last day of the selective, interns again meet with a member of the genetics faculty to review their experience and take the post-test. The post-test contains the same questions as the pretest (Supplementary Appendix A). The correct answers to the post-test are reviewed at this time as well as any other questions that the resident has pertaining to any of their experiences on the selective.

\section{Rotation evaluation}

On the last day of the selective, interns also complete a rotation evaluation (Supplementary Appendix D) consisting of 12 questions using Likert scales. Interns also have space to 
write any comments regarding their clinical experiences or the provided educational content. While positive comments are welcomed, interns are also encouraged to give verbal and written constructive feedback so that the new rotation can continue to be improved for future participants in an iterative fashion.

\section{Analysis}

Data were analyzed in aggregate as a cohort with no names attached to the pre- and post-tests, logbooks, or evaluations, so that these could remain anonymous to the analyst. Average pretest and post-test scores were compared using a paired $t$-test. A Chi-square test was used to determine statistical differences between each question on the pre- and post-test. The overall evaluation of the rotation was calculated by averaging responses to each question. Mean averages were also calculated for number of patients and different diagnoses seen during the rotation.

\section{RESULTS}

Fifty interns (approximately 50\% of eligible trainees) have thus far participated in the genetics selective. Forty participants were categorical pediatric residents, five were pediatric neurology residents, three were pediatric anesthesiology residents, and there was one resident each from the pediatrics/genetics and medicine/pediatrics training tracks.

Qualitative comments regarding the selective highlighted several common themes. Rotators thought the experience provided a unique exposure to genetics with a variety of patient experiences, commenting that it was "a great 'window' into genetics," "a great introduction to genetics, especially as it relates to being a general pediatrician," and "a great exposure to the process of a genetic work-up and specific rare diagnoses." Others commented on how the rotation introduced resources and concepts that could be useful in the future, saying "I gained a better understanding of what resources I can use going forward for genetics concerns" and "required readings were helpful in explaining features and genetic testing." Another common theme throughout written feedback was that participants wished they had more days on the rotation.

Quantitative evaluations completed by 45 participants revealed an average score of 4.4 out of 5 . This score has been consistent among each intern class of participants.

Forty-four participants completed both the pre- and posttest. The average pretest score was $2.5 / 5(50 \%)$ and the average post-test score was $4.3 / 5(86 \%)$. These scores were statistically significant in a paired $t$-test $(p<0.00001)$. The questions that showed the most improvement in correct answers involved knowing indications for chromosomal microarray $(37 \%$ vs. $86 \%) \quad(p<0.00001)$, resources for abnormal newborn screening ( $23 \%$ vs. $88 \%)(p<0.00001)$, and possible outcomes of microarrays and clinical exome sequencing $(16 \%$ vs. $84 \%) \quad(p<0.00001)$. Questions that showed no significant improvement involved knowing appropriate genetic counseling language (95\% vs. 100\%)
Table 1 Variety of patient experiences.

\begin{tabular}{lll} 
& Average & Range \\
\hline Number of patient experiences & 9.7 & $2-18$ \\
\hline Number of diagnoses seen & 3.8 & $1-6$ \\
\hline Diagnosis = inborn error of metabolism & 1.8 & $0-5$ \\
\hline $\begin{array}{l}\text { Diagnosis = dysmorphic feature/congenital } \\
\text { anomaly including skeletal dysplasia }\end{array}$ & 2.8 & $0-7$ \\
$\begin{array}{l}\text { Diagnosis = connective tissue disease } \\
\text { Diagnosis = developmental or neurological } \\
\text { disorder }\end{array}$ & 2.8 & $0-7$ \\
$\begin{array}{l}\text { Diagnosis = other (e.g., cancer syndrome, } \\
\text { immunological disorder) }\end{array}$ & 0.5 & $0-4$ \\
\hline
\end{tabular}

Table 2 Independent application of clinical skills.

\begin{tabular}{|c|c|}
\hline $\begin{array}{l}\text { Number of participants who performed clinical skills } \\
\text { independently }\end{array}$ & $19 / 40(48 \%)$ \\
\hline Skill = genetic focused history & $18 / 40(45 \%)$ \\
\hline Skill = genetic focused physical exam & $19 / 40(48 \%)$ \\
\hline Skill $=$ pedigree construction & $18 / 40(45 \%)$ \\
\hline
\end{tabular}

$(p=0.5)$ and specific features of connective tissues diseases ( $72 \%$ vs. $77 \%)(p=0.6)$.

Forty participants provided a completed logbook that indicated they saw on average nine patients throughout their week (range: 2-18) and an average of four diagnoses (range: 1-6) (Table 1). Ranges are wide because two participants had less than a week of in-person time on the selective due to extenuating circumstances. About half of participants (48\%) indicated that they independently performed genetically relevant clinical skills (Table 2).

\section{DISCUSSION}

Our evaluation of the genetics selective suggests that it was a positive experience for the interns who participated and resulted in an improvement in knowledge regarding several genetics issues. Because of the completion of the Human Genome Project in 2003 and ongoing discoveries in medical genetics, the practice of medicine as it relates to diagnosis and management has an increasing capability to become much more individualized. The limiting factor in integrating genetics into clinical care has been lack of knowledge and training on the subject matter. ${ }^{10}$ The American Academy of Pediatrics (AAP) has worked to increase genetic literacy through their Genetics in Primary Care Institute (GPCI) ${ }^{11}$ There is however, no standard genetics training or mandatory curriculum in pediatric training programs, but such a curriculum is needed so that future pediatricians are able to confidently deliver care as it pertains to genetic medicine.

In 2010, a workshop convened by the National Institutes of Health, the Centers for Disease Control and Prevention, and the Health Resources and Services Administration focused on how to educate primary care physicians to better enable them 
to deliver genetic-related care. They cited developing targeted curricula for residency training programs as a practical strategy to educate primary care physicians. ${ }^{12}$ The genetics selective implemented for the trainees in the Johns Hopkins pediatric residency program provides interns with an opportunity to experience such a structured curriculum during their training. A prior study showed that obstetrics and gynecology residents have increased knowledge on women's health genetics after three interactive modules, but this is the first report that includes data on the knowledge and skills gained by doing a rotation in genetics with actual clinical encounters. ${ }^{13}$ This report also provides a model so that similar experiences can be replicated at other institutions.

Based on the results described above, the experiences of the interns who have participated in the genetics selective thus far suggest success in the first three years of the selective with positive qualitative feedback and quantitative assessments. A future direction of this study may include further data collection from residents at the end of their training. Analysis could be done to assess retention of the knowledge they learned and to ascertain if it impacted the way they practice medicine, in addition to determining if participation influenced their future career trajectory.

As the genetics selective continues, materials such as the scavenger hunt and the provided articles will be updated as indicated based on the emergence of new relevant topics in genetics as they relate to general pediatrics. Emphasis will remain on enabling interns to have meaningful patient encounters as part of a multidisciplinary approach to clinical care. Residents should learn the indications for a referral to genetics and also be able to order appropriate standard genetic testing (i.e., microarray) after providing informed consent on possible outcomes of such genetic testing. They will continue to use resources to learn to evaluate and manage patients with suspected or known genetic conditions.

Current limitations of this rotation, and therefore this education report, are that this is only a single institution experience with a limited number of participants. Since participants are only those interns who select the subspecialty option for the selective, and not those who select the primary care selective, there is an inherent bias in favor of subspecialty medicine among participants and less exposure to genetics for those interns more likely to go into primary care. Additionally, only one of the two weeks of their selective time is spent within genetics. The time spent, however, is well utilized given the robust nature of the Genetics Department at Johns Hopkins Medicine, a tertiary care center with multiple subspecialty genetics clinics run by nine practicing clinical geneticists.

Nationally, there should be a movement to make training in medical genetics a requirement in ACGME-accredited pediatric programs so that the existing deficiency in knowledge of medical genetics can be remedied. A similar curriculum could be offered at other institutions that have a genetics department with ample opportunities for clinical experiences for residents, but this may be difficult for smaller programs without such resources. Programs that have fewer resources could still implement a formal curriculum using alternative educational methods, such as didactic conferences or web-based modules. ${ }^{14}$

\section{SUPPLEMENTARY INFORMATION}

The online version of this article (https://doi.org/10.1038/s41436019-0723-7) contains supplementary material, which is available to authorized users.

\section{DISCLOSURE}

The authors declare no conflicts of interest.

Publisher's note Springer Nature remains neutral with regard to jurisdictional claims in published maps and institutional affiliations.

\section{REFERENCES}

1. Collins FS. Medical and societal consequences of the Human Genome Project. N Engl J Med. 1999;341:28-37.

2. Jenkins J, Blitzer $M$, Boehm K, et al. Core competencies in genetics essential for all health professionals. Genet Med. 2001;3:155-159.

3. Accreditation Council for Graduate Medical Education. Program requirements for graduate medical education in pediatrics. 30 June 2017. https://www.acgme.org/Portals/O/PFAssets/ProgramRequirements/ 320_pediatrics_2017-07-01.pdf.

4. Harvey EK, Fogel CE, Peyrot M, Christensen KD, Terry SF, Mclnerney JD. Providers' knowledge of genetics: a survey of 5915 individuals and families with genetic conditions. Genet Med. 2007;9:259-267.

5. Saul RA, Trotter T, Sease K, Tarini B. Survey of family history taking and genetic testing in pediatric practice. J Community Genet. 2017:8:109-115.

6. Peabody J, DeMaria L, Tamandong-LaChica D, Florentino J, Acelajado MC, Burgon T. Low rates of genetic testing in children with developmental delays, intellectual disability, and autism spectrum disorders. Glob Pediatr Health. 2015;2:2333794X15623717.

7. Nguyen J, Lemons J, Crandall S, Northrup H. Efficacy of a medical genetics rotation during pediatric training. Genet Med. 2016;18:199-202.

8. Rubanovich CK, Cheung C, Mandel J, Bloss CS. Physician preparedness for big genomic data: a review of genomic medicine education initiatives in the United States. Hum Mol Genet. 2018;27:R250-R258.

9. Thatcher J, Canfield P, Bauer L, Griffith BN. Pedigree analysis: a teambased learning activity. MedEdPORTAL. 2017;13:10557.

10. Secretary's Advisory Committee on Genetics, Health, and Society. Genetics education and training report. 2011. https://www.eshg.org/fileadmin/ www.eshg.org/documents/committees/SACGHS_education_report_2011. pdf

11. Saul RA. Genetic and genomic literacy in primary care. Pediatrics. 2013;132 Suppl 3:S198-S202.

12. Kemper AR, Trotter TL, Lloyd-Puryear MA, Kyler P, Feero WG, Howell RR. A blueprint for maternal and child health primary care physician education in medical genetics and genomic medicine: recommendations of the United States secretary for health and human services advisory committee on heritable disorders in newborns and children. Genet Med. 2010;12:77-80.

13. Dotters-Katz S, Hocutt G, Osborne CM, Hardisty EE, Demmer L, Vora N. Development, implementation, and assessment of a genetics curriculum across institutions. AJP Rep. 2016;6:e372-e377.

14. Riegert-Johnson DL, Korf BR, Alford RL, et al. Outline of a medical genetics curriculum for internal medicine training programs. Genet Med. 2004;6:543-547. 